Annalisa Andreoni

\title{
Sull'esemplarità del caso di Margherita Costa negli studi di letteratura italiana
}

\begin{abstract}
Annalisa Andreoni, coordinatrice del Gruppo di Studi delle donne nella letteratura italiana dell'Associazione degli Italianisti, apre i lavori del Convegno Margherita Costa, poetessa virtuosa (L'Aquila, 12 aprile 2021).
\end{abstract}

The e-journal «altrelettere» is hosted at the URL: http://www.altrelettere.uzh.ch, in accordance with the Open Access Policy of the University of Zurich. Please cite this article as follows: A. ANDREONI, Sull'esemplarità del caso di Margherita Costa negli studi di letteratura italiana, in «altrelettere», (2021), pp. 8-12, DOI: 10.5903/al_uzh-50. (C) This article is licensed under a Creative Commons Attribution 2.5. Switzerland (CC BY-NC-ND 2.5). Please read the license terms on the website: http://creativecommons.org/licenses/by-nc-nd/2.5/ch/deed.en 


\section{Introduzione}

Ho accolto con molto piacere l'invito delle organizzatrici di questo convegno, Daniela De Liso e Valeria Merola, a portare il saluto del gruppo di lavoro di "Studi delle donne nella letteratura italiana" dell'AdI - Associazione degli italianisti. ${ }^{1}$ Ringrazio le organizzatrici per l'invito e mi complimento per questo convegno che, per l'argomento e per il respiro internazionale, è davvero un'incarnazione dello spirito che anima il nostro gruppo di lavoro.

Il gruppo di "Studi delle donne" è attivo ormai da sette anni all’interno dell'AdI, e mi piace ricordare che è stato il primo gruppo di lavoro a nascere all'interno dell'Associazione. Quando feci questa proposta all'allora presidente Guido Baldassarri, non esistevano strutture di questo tipo all'interno dell'AdI, ma egli accettò subito con molta apertura la costituzione del gruppo. La formula si è rivelata felice, e adesso sono vari i gruppi operanti all'interno dell'Associazione: da quello su Dante a quello sul Rinascimento a quello sul Risorgimento, solo per citarne alcuni.

Il nostro Gruppo è sorto dunque nel 2014 con l'intenzione di costruire una rete tra i vari gruppi di ricerca che lavorano sulla produzione letteraria femminile in Italia, e per creare un luogo a cui potessero fare riferimento anche singole studiose e singoli studiosi che autonomamente lavorino su questi temi. Tra gli scopi principali vi è stato fin da subito quello di offrire la possibilità di far circolare nella nostra rete le notizie sulle iniziative che si stanno organizzando e sulle pubblicazioni che si producono.

A questo scopo raccogliamo e diffondiamo sulla nostra pagina all'interno del sito dell'AdI le notizie che ci arrivano. Si tratta di un obiettivo che abbiamo avuto fin dall'inizio della nostra attività: porci cioè al servizio della comunità degli italianisti, rendere più visibili gli studi nel campo della letteratura femminile, che a livello nazionale sono numerosi e sono di qualità.

L'altro obiettivo che abbiamo perseguito in questi anni, più ambizioso, è stato quello di conquistare per gli studi sulla letteratura femminile uno spazio di legittimità all'interno dell'Associazione degli italianisti, convinte e convinti come siamo che la storia della letteratura italiana debba in gran parte essere riscritta tenendo conto anche della produzione delle autrici, le quali sono state troppo spesso pregiudizialmente espunte dalla storiografia letteraria prevalente. Il nostro intento, semplice ma ambizioso, è di arrivare alla 
costituzione di un nuovo canone letterario, che includa autori e autrici, ognuno naturalmente con la propria caratura e grandezza, ma senza più pregiudiziali espunzioni di genere.

E con questo obiettivo abbiamo organizzato ogni anno, all'interno dei Congressi dell'AdI, delle sessioni dedicate specificamente alle autrici, sia sessioni parallele aperte alle proposte dei partecipanti sia sessioni plenarie su invito. Colgo l'occasione di essere qui tra voi per annunciare che stiamo organizzando, per il 15 e il 16 dicembre 2021, un convegno che cercherà di aggredire proprio il canone della narrativa del Novecento. Sarà un convegno online aperto alle proposte esterne, ci sarà un invito a presentare relazioni incentrate specificamente su singole opere narrative di autrici del Novecento che meritano di essere incluse nel canone. Partiamo dalla narrativa, ma abbiamo intenzione di proseguire poi con il canone della poesia del Novecento, del teatro e così via.

Ma venendo all'occasione di oggi, a questo bel convegno che avete organizzato, ebbene, Margherita Costa è davvero un caso esemplare di pregiudiziale espunzione dalla storiografia letteraria di una figura dotata di voce poetica originale, autonoma e molto feconda, e che si muove in ambito europeo, tra l'Italia e la corte francese. Un'espunzione pregiudiziale tanto più bruciante in quanto avvenuta, prevalentemente e palesemente, direi, per pregiudizi sulla condotta di vita della poetessa.

Negli anni più recenti c'è stato finalmente un interesse a livello internazionale, ma non si può dimenticare come fino a non molti anni fa - parlo della metà degli anni Ottanta - all'interno di un'opera canonica della storiografia come il Dizionario Biografico degli Italiani la voce dedicata a Margherita Costa ancora fosse segnata da giudizi paternalistici e moralistici legati proprio alla condotta di vita dell'autrice, e ciò nonostante fosse a firma di uno studioso autorevole, riconosciuto, importante del Seicento come Martino Capucci. Il problema, a mio parere, non riguarda i singoli, ma più generale l'approccio alla produzione letteraria femminile.

Nella voce si legge, per esempio, a proposito della Tromba di Parnaso, e in riferimento a una caratteristica che in un canzoniere maschile sarebbe stata considerata degna di essere studiata e valutata - e cioè le numerose dediche a personaggi nobili e potenti - che si tratterebbe di «particolare scialo di dediche 
a personaggio di levatura europea» (CAPUCCI 1984, 232). E si legge, ancora, che Margherita Costa corrispose alla protezione del Mazzarino «con frequenti e smaccati omaggi poetici [...] dove ancora una volta si adopera, in modi più patetici che furbeschi, a cercar benevolenza presso i potenti» (ibidem). Leggere queste parole oggi fa una certa impressione e davvero convince della necessità di leggere finalmente le nostre autrici del passato con occhi liberi da pregiudizi.

Quindi ancora una volta grazie per aver organizzato questo convegno: vi auguro buon lavoro e spero che si possano vedere presto pubblicati anche gli atti e di questa giornata di studio. 


\section{Note}

1 Per informazioni sul Gruppo di lavoro "Studi delle donne nelle letteratura italiana" dell'AdI-Associazione degli Italianisti, rimando a seguente link: https://www.italianisti.it/gruppidi-lavoro/studi-delle-donne-e-di-genere-nella-letteratura-italiana/presentazione

\section{Bibliografia}

\section{CAPUCCI 1984:}

Martino CAPUCCI, Costa, Margherita, in Dizionario biografico degli italiani, Roma, Enciclopedia Treccani, 1984, vol. 30, pp. 232-34. 